\title{
Wide Absorption Spectrum Measuring Methods by DFB-LDs in Water Vapor Detection System
}

\author{
Y. N. LIU ${ }^{1}$, J. CHANG ${ }^{1 *}$, J. LIAN ${ }^{1}$, Q. WANG ${ }^{1}$, G. P. LV ${ }^{1}$, W. J. WANG ${ }^{1}$, Z. LIU ${ }^{1}$, \\ X. Z. LIU $^{2}$, S. S. ZHANG ${ }^{1}$, Z. L. WANG ${ }^{1}$, S. JIANG ${ }^{1}$, C. G. ZHU ${ }^{1}$, \\ W. WEI ${ }^{1}$, and B. N. SUN ${ }^{1}$ \\ ${ }^{1}$ School of Information Science and Engineering and Shandong Provincial Key Laboratory of Laser Technology and \\ Application, Shandong University, Jinan, 250100, China \\ ${ }^{2}$ Institute of Automation, Shandong Academy of Sciences, Jinan, 250014, China \\ *Corresponding author: J. CHANG E-mail: changjun@sdu.edu.cn
}

\begin{abstract}
Two measuring methods of the wide absorption spectrum by distributed feedback laser diodes (DFB-LDs) are presented in detecting the water vapor absorption line. One is the subsection scanning method, and it takes advantage of the wide spectrum tuning range by the temperature modulation and fast spectrum tuning speed by current modulation. Specifically, this method is realized by dividing a target spectral region into several sections which correspond to the specific temperature of DFB-LD, scanning every section by current modulation for hundreds times, and averaging the data to raise the signal to noise ratio (SNR), then combining all sections to get the whole spectrum. An accuracy of $10 \mathrm{ppmv}$ had been obtained in the measurement of water vapor with a $10-\mathrm{cm}$ path length by this method. Another is data fitting method, based on the absorption line-shape function; the absorption line can be described by fitting with partial measured data. The fitting absorption line was fitted well with the measured data, and the square of correlation coefficient (R-square) was no less than 0.99 .
\end{abstract}

Keywords: DFB-LD, wide spectrum, subsection scanning, data fitting, water vapor detection

Citation: Y. N. LIU, J. CHANG, J. LIAN, Q. WANG, G. P. LV, W. J. WANG, et al., "Wide Absorption Spectrum Measuring Methods by DFB-LDs in Water Vapor Detection System," Photonic Sensors, 2014, 4(3): 230-235.

\section{Introduction}

$\mathrm{SF}_{6}$ performs excellent in insulation and arc-extinction, given such advantages, $\mathrm{SF}_{6}$ gas insulated switchgears (GIS) are widely applied in the electrical power systems. In these applications, it is vital to ensure the $\mathrm{SF}_{6}$ purity, because trace water vapor inside could affect the insulation and the resultants corrode the equipments, both could cause serious accidents $[1,2]$. Therefore, trace water vapor monitoring is of great necessity in this field. Among various detection methods, tunable diode laser absorption spectroscopy (TDLAS) has proven an effective method for trace gases concentration measurement, and its outstanding features include the high resolution, accuracy, sensitivity, and fast response [3]. The traditional TDLAS technique usually drives a distributed feedback laser diode (DFB-LD) scanning over a whole gas absorption line and then gets the peak value in the electronic method, only in a low gas pressure condition it works well, where the absorption line is not

Received: 3 November 2013/ Revised version: 2 December 2013

(C) The Author(s) 2013. This article is published with open access at Springerlink.com

DOI: $10.1007 / \mathrm{s} 13320-013-0152-1$

Article type: Regular 
substantially broadened by pressure [4]. Whereas, in $\mathrm{SF}_{6}$ gas insulated switchgears, the gas pressure can usually be several atmospheres (atm). Take the $1368.597 \mathrm{~nm}$ absorption line of water vapor as an example. In the Lorentzian profile case, the full width at half maximum (FWHM) is proportional to the atmospheric pressure. At $6 \mathrm{~atm}, 296 \mathrm{~K}$, the FWHM can even reach up to $218 \mathrm{pm}$, and the whole line extends to $925 \mathrm{pm}$ when taking $5 \%$ of the peak value as the baseline. In our system, the adopted DFB-LD (such as WSLS-137010C1424-20) wavelength tuning range is less than $320 \mathrm{pm}$ under current modulation (corresponding to a $60 \mathrm{~mA}$ current range), obviously, it is impossible to scan the whole absorption line by current modulation; temperature modulation can tune a wider range of $1800 \mathrm{pm}$ (corresponding to a $20-^{\circ} \mathrm{C}$ temperature range), but its modulation frequency is only about $1 / 30 \mathrm{~Hz}$, which is too low that common filters can hardly fit it, furthermore, the effective average process would cost a overlong time, thus temperature modulation is not a proper way either.

In this paper, the solutions are proposed in two ways to cope with this task: one way is subsection scanning, and it is realized by dividing a target spectral region into several sections, then scanning each section by current modulation for hundreds scans, finally according to unique points "sewing" all of the sections up in order; the other way is fitting, if the line profile function is given, the whole absorption line could be fitted according to a few correctly measured values pairs on the line.

\section{Methods design}

\subsection{Subsection scanning method}

The emission wavelength of a DFB-LD can be tuned by modulating the operating temperature or injection current [5]. Current modulation is fast but its tuning range is restricted; temperature modulation can obtain a wider spectral region but limited by its modulation frequency in applications. In essence, we obtained an intending wavelength with a DFB-LD by matching a proper temperature and a proper current value through circuits. Based on this fact, we proposed a subsection scanning method. We supposed that the measured spectral range was within the temperature tuning range. Firstly, to divide such spectral region into several sections, the wavelength range of one section is equal to the tuning range of DFB-LD in current modulation. Then, to scan every section in turn by setting different operating temperatures of the DFB-LD. Finally, to integrate all sections by data processing according to some feature points or synchronization signals.

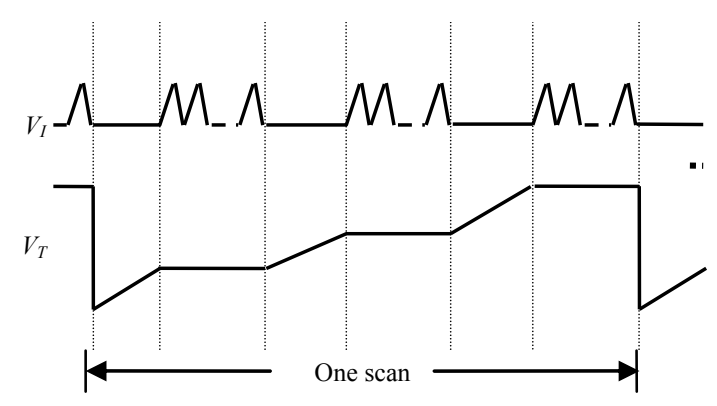

(a) Drive signal of LD

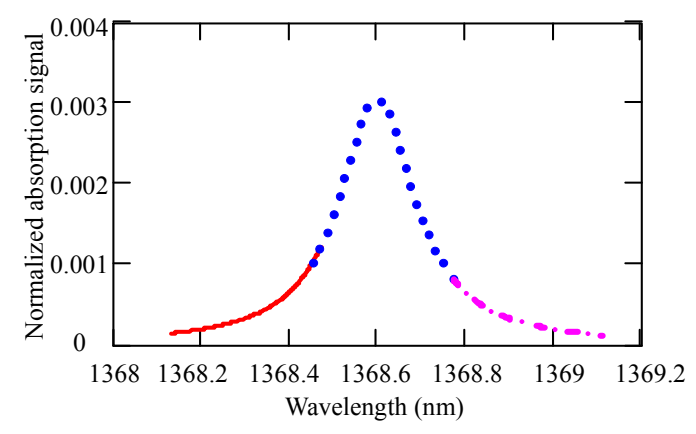

(b) Integrated absorption line $(1368.597 \mathrm{~nm})$ at $6 \mathrm{~atm}$

Fig. 1 Scanning simulation of $1368.597 \mathrm{~nm}$ absorption line at $6 \mathrm{~atm}$.

Based on the subsection scanning method, a scanning simulation about the water vapor absorption line at $1368.597 \mathrm{~nm}$ is given in Fig. 1. At $6 \mathrm{~atm}, 296 \mathrm{~K}$, the absorption line was broadened to about $925 \mathrm{pm}$ when taking $5 \%$ of the peak value as the baseline, and this spectral range was within the temperature tuning range of $1800 \mathrm{pm}$ and obviously beyond the current tuning range, $320 \mathrm{pm}$. The broadening absorption line was divided into 3 sections, every section was $320 \mathrm{pm}$, and the 
adjacent sections overlapped each other by about $17 \mathrm{pm}$. Figure 1(a) shows the drive signal of LD, $V_{T}$ represents the controlling signal of the operating temperature, and $V_{I}$ represents that of the drive current. Figure 1(b) is the final integrated absorption line, three sections are described with different lines, and each of them is scanned by current modulation.

\subsection{Data fitting method}

The basic theory of direct absorption spectroscopy is the Beer-Lambert law. The intensity of monochromatic laser radiation transmitted through a gas cell containing absorbing specie is related as follows [6]:

$$
\begin{gathered}
I_{t}(v)=I_{0} \exp [-k(v) L] \\
k(v)=P S(T) g\left(v-v_{0}\right) N
\end{gathered}
$$

where $I_{t}(v)$ is the transmitted intensity, $I_{0}$ is the incident intensity, $k(v)\left(\mathrm{cm}^{-1}\right)$ is the spectral absorption coefficient, and $L(\mathrm{~cm})$ is the length of the gas absorption length; $P(\mathrm{~atm})$ is the total gas pressure, $S(T)\left(\mathrm{cm}^{-2} \cdot \mathrm{atm}^{-1}\right)$ is the line strength at temperature $T(K), g\left(v-v_{0}\right)(\mathrm{cm})$ is the line shape function, and $N$ is the mole fraction of target gas.

According to (1) and (2), the line shape function $g\left(v-v_{0}\right)$ can reflect the absorption coefficient change, therefore, the absorption line can be obtained by fitting according to the line-shape function $g\left(v-v_{0}\right)$ theoretically [7]. Three typical line shapes are Gaussian, Lorenz, and Voigt profiles, respectively. They dominate at different pressures, the Lorenz profile agrees well with the Voigt profile when $P \geq 0.4 \mathrm{~atm}$, and the Gauss profile is in good agreement with the Voigt profile when $P \leq 0.005 \mathrm{~atm}$ [8]. In practical applications, gas pressure usually is the atmosphere level (usually $P \geq 1 \mathrm{~atm}$ ), thus, the Lorenz profile should be the appropriate absorption line-shape. When the gas pressure increases, the absorption line would be broadened, and the limited wavelength tuning range of drive current modulation could not overlap the absorption line completely. In this condition, the fitting curve (based on the line-shape function) can be used to describe the entire absorption line.

\section{Experimental setup and results}

\subsection{Experimental setup}

The schematic diagram of the detection system is shown in Fig. 2. A DFB-LD (WSLS$137010 \mathrm{C} 1424-20)$ at $1370 \mathrm{~nm}$ was chosen as the light source. An ARM7 (LTC1758, NXP, Netherlands) was used to control the laser operating temperature via a temperature control chip (LTC1923, Linear Technology, USA), and another ARM7 generated a trapezoidal wave to drive the DFB-LD scanning over the $320 \mathrm{pm}$ spectrum with a wavelength resolution of $1.5 \mathrm{pm}$. For subsection scanning, the two ARMs dominate alternately as the program designed. In the experiment, a dual-beam structure was adopted, and pure $\mathrm{N}_{2}$ with a purity of 99.999\% served as the carrier gas. The concentration of water vapor in pure $\mathrm{N}_{2}$ was varied by a low dew point generator (3900, THUNDER, the USA), and the resolution could be $0.10{ }^{\circ} \mathrm{C}$. And a high performance dew point hygrometer (S4000TRS, MICHELL, UK), with a resolution of $0.10{ }^{\circ} \mathrm{C}$ dew point, was used as a standard instrument to calibrate the system.

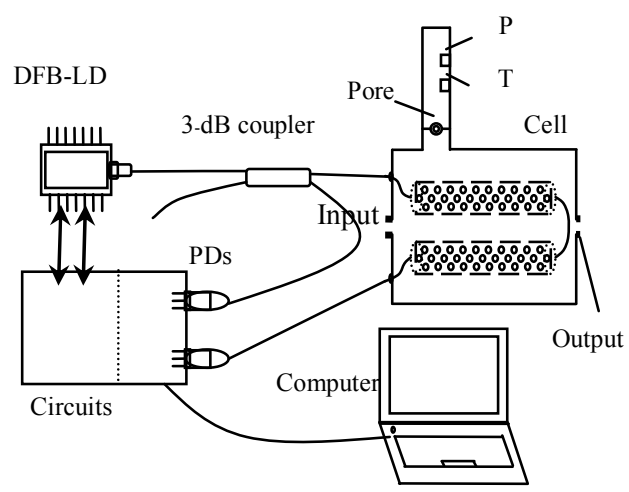

Fig. 2 Schematic diagram of the detection system.

\subsection{Application of subsection scanning in the dual-peak measuring method}

A so called dual-peak method of water vapor concentration detection was proposed by our group [9]. In this method, the difference between two peak values of the adjacent absorption lines of water 
vapor (at $1367.862 \mathrm{~nm}$ and $1368.597 \mathrm{~nm}$, respectively) had proven a linear relation with the concentration of water vapor [9]. In other words, the difference value has nothing to do with the integrity of the absorption lines, as long as the two peaks can be measured, and the water vapor can be detected. To scan such a wide spectrum, temperature modulation was adopted in [9], because of the slow scanning speed, it is limited in applications. In order to raise the SNR, we used the subsection scanning method to scan the two absorption peaks. The wavelength space was $735 \mathrm{pm}$ between $1367.862 \mathrm{~nm}$ and $1368.597 \mathrm{~nm}$, and the DFB-LD had a wavelength tuning range of $320 \mathrm{pm}$ by the drive current. Ignoring the useless middle region between the two absorption lines, the tuning range consisted of two sections, corresponding to the absorption lines at $1367.862 \mathrm{~nm}$ and $1368.597 \mathrm{~nm}$, respectively. In the calibration process, the dew point generator 3900 generated 3 dew points at $-55^{\circ} \mathrm{C},-40^{\circ} \mathrm{C}$, and $-20{ }^{\circ} \mathrm{C}$, respectively. Synchronously, we measured the water vapor concentration value in $\mathrm{N}_{2}$ with S4000 and the signal voltage with the setups in Section 3.1 recorded as $21.47 \mathrm{ppmv}, 140.2 \mathrm{ppmv}$, $1039.7 \mathrm{ppmv}$, and $147 \mathrm{mV}, 395 \mathrm{mV}, 2274 \mathrm{mV}$, respectively. Thus, the relation between the vapor concentration $(y)$ and signal voltage $(x)$ can be given as follows:

$$
y=0.4787 x-48 .
$$

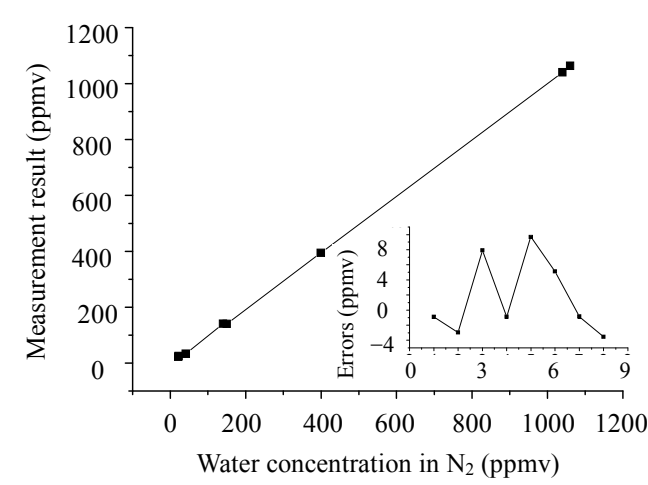

Fig. 3 Measurement result and measuring errors.

Based on the above relation, we had an 8-point test, and the measurement result is shown in Fig. 3.
The ambient temperature and the gas pressure inside the gas cell were the same with the calibration process, still remained $296 \mathrm{~K}$ and the normal atmosphere. The measurement result was well proportional to the real value from $21.47 \mathrm{ppmv}$ to 1060 ppmv. And the measuring error between the real value and the measured value was within 10 ppmv, better than $20 \mathrm{ppmv}$ in [9], in which the LD was tuned by temperature modulation.

\subsection{Data fitting in measuring broadening absorption line}

The wavelength tuning range of our LD under current modulation was no more than $320 \mathrm{pm}$. In the high pressure case, the absorption line would be broadened. For $1368.597 \mathrm{~nm}$ absorption line at $6 \mathrm{~atm}$, $296 \mathrm{~K}$, the FWHM was about $218 \mathrm{pm}$, and the whole line absolutely could not be overlapped with $320 \mathrm{pm}$. Figure 4(a) gives the measurement result at $6 \mathrm{~atm}$ by current modulation, only partial absorption line was scanned, and fortunately, it still remained the Lorenz line-shape with an R-square of 0.99. Figure 4(b) just gives an example of data fitting for the absorption line in our design. In the figure, the measured partial absorption line was plotted in the solid line, and the whole absorption line was described by a Lorenz fitting curve in the dotted line. This fitting curve was obtained by 80 data points, selected from the 1021 data points measured at $6 \mathrm{~atm}$ and shown in the inset in Fig. 4(b). There is a relatively great difference between the fitting result and the measured data at the falling edge of the absorption line, and this may be caused by filters, because broadening could increase the frequency components in the absorption line. To some degree, filters response differently to different frequencies.

There are two main reasons of data fitting with fewer data points: one is to reduce the data volume and shorten time in data processing, and the other is to exclude the data around the peak region, which even contains the water absorption signal in some other components in the light path and such water 
vapor is usually free from gas pressure variation outside.

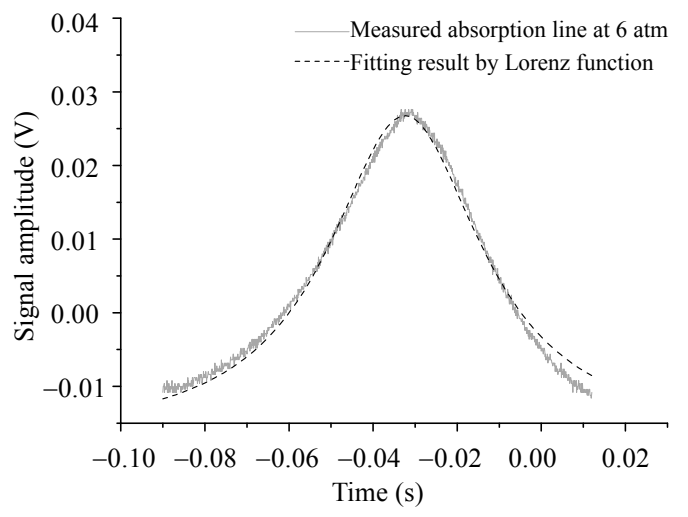

(a) Fitting with all measured data

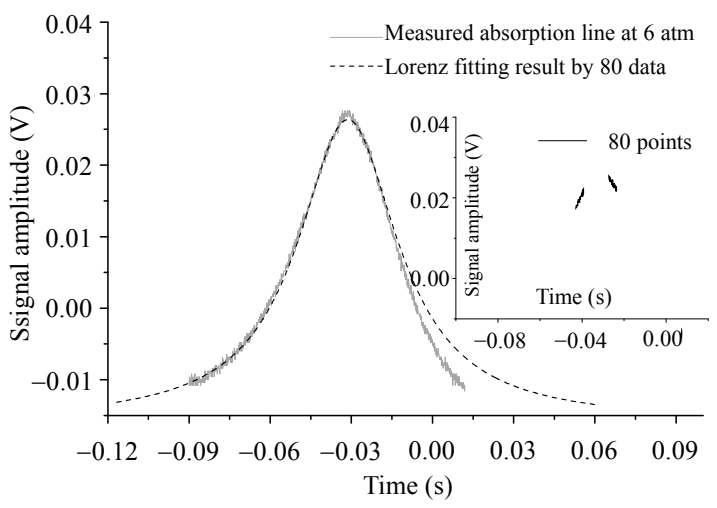

(b) Fitting with partial measured data

Fig. 4 Fitting results of $1368.597 \mathrm{~nm}$ absorption line at $6 \mathrm{~atm}$ with different point numbers.

\section{Discussion}

The LD used in the experiments had a temperature modulation frequency of $0.03 \mathrm{~Hz}$. It is of great difficulty to design such a low-pass filter, and a serious signal distortion would be caused by a low-pass filter even with a higher cutoff frequency [10]. Average process is unpractical due to the time cost. In subsection scanning, every section had a number of scans, thus, noise could be effectively suppressed by average.

For data fitting, it is simple and fast, but it should be cautious in data selection, and the instability data region should be avoided, such as the top region of the signal in Fig. 4. Because there is other water absorption in optical components, such absorption would be superimposed on the measured signal and usually free from gas pressure variation, when gas pressure in gas cell varies, and the related absorption line-shape changes correspondingly, while the water inside the optical components is not affected, which could lead to a deformation at the top of signal.

\section{Conclusions}

In this article, we propose two measuring methods of the wide absorption spectrum by commercially available DFB-LDs for water vapor concentration detection. The subsection scanning method combines the advantages of the temperature and drive current modulations. Based on the dual-peak method, an accuracy of 10 ppmv had been obtained in the water vapor concentration detection with a $10-\mathrm{cm}$ path length. Such a high resolution was benefit from the high SNR achieved by the average process. A data fitting method was also applied to measure the water absorption line. With the given Lorenz profile function, the absorption line at $6 \mathrm{~atm}$ was obtained with a fewer number of measured data, thus, this method can be very efficient, and it is of great potential for industrial applications.

\section{Acknowledgment}

This work was supported by Natural Science Foundation of China (60977058 \& 61205083), Independent Innovation Foundation of Shandong University (IIFSDU2010JC002 \& 2012JC015), the key technology projects of Shandong Province (2010GGX10137), and promotive research fund for excellent young and middle-aged scientists of Shandong Province (BS2010DX028).

Open Access This article is distributed under the terms of the Creative Commons Attribution License which permits any use, distribution, and reproduction in any medium, provided the original author(s) and source are credited.

\section{References}

[1] S. C. Zhang, Q. Wang, Y. Zhang, F. J. Song, K. Chen, 
G. Q. Chou, et al., "Water vapor detection system based on scanning spectra," Photonic Sensors, 2012, 2(1): 71-76.

[2] Y. Zhang, J. Chang, Q. Wang, S. C. Zhang, and F. J. Song, "The theoretical and experimental exploration of a novel water-vapor concentration measurement scheme based on scanning spectrometry," 2011 International Conference on Electronics and Optoelectronics, China, Jul. 29-31, 2011.

[3] L. M. Wang, Y. J. Zhang, H. B. Li, Y. Zhou, Y. He, and W. Q. Liu, "Study on long distance transmission technique of weak photocurrent signal in laser gas sensor," Chinese Optics Letters, 2012, 10(4): 042802.

[4] P. Werle, "A review of recent advances in semiconductor laser-based gas monitors," Spectrochimica Acta Part A: Molecular and Biomolecular Spectroscopy, 1998, 54(2): 197-236.

[5] V. Weldon, D. McInerney, R. Phelan, M. Lynch, and J. Donegan, "Characteristics of several NIR tuneable diode lasers for spectroscopic based gas sensing: a comparison," Spectrochimica Acta Part A: Molecular and Biomolecular Spectroscopy, 2006, 63(5): 1013-1020.

[6] X. Liu, J. B. Jeffries, and R. K. Hanson, "Measurements of spectral parameters of watervapour transitions near 1388 and $1345 \mathrm{~nm}$ for accurate simulation of high-pressure absorption spectra," Measurement Science and Technology, 2007, 18(5): 1158-1194.

[7] W. G. Chen, Y. X. Yun, C. Pan, and C. X. Sun, "Analysis of infrared absorption properties of dissolved gases in transformer oil," Proceeding of the Chinese Society for Electrical Eegineering, 2008, 28(16): 148-153.

[8] J. Chang, G. P. Lv, G. Q. Zhou, K. Chen, Y. Zhang, Z. L. Wang, et. al., "Application of wavelength scanning for measuring water vapour concentration by distributed laser diode," Procceeding of 5th Photonic Fiber and Crystal Devices: Advances in Materials and Innovations in Device Applications, USA, Aug. 21, 2011.

[9] Q. Wang, J. Chang, C. G. Zhu, C. Li, F. J. Song, Y. N. Liu, et al., "Detection of water vapor concentration based on differential value of two adjacent absorption peaks," Laser Physics Letters, 2012, 9(6): 421-425.

[10] J. Chang, Q. J. Huang, X. Z. Liu, K. Chen, H. T. Chang, G. P. Lv, et al., "Noise investigation and signal process in fiber optic gas sensor with wavelength modulation of the DFB laser diode," Proceeding of 6th Photonic Fiber and Crystal Devices: Advances in Materials and Innovations in Device Applications, USA, Aug. 12, 2012. 